
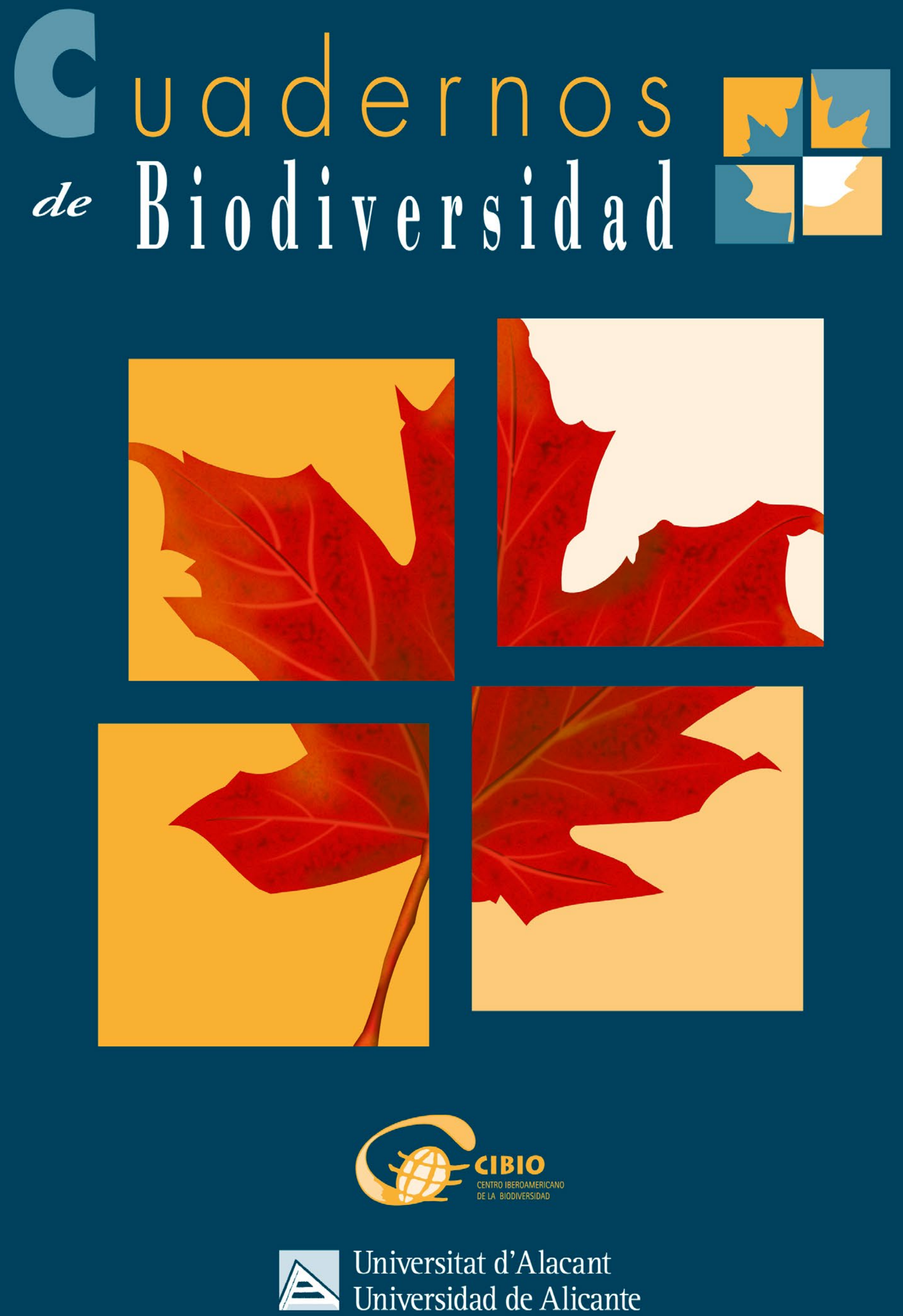


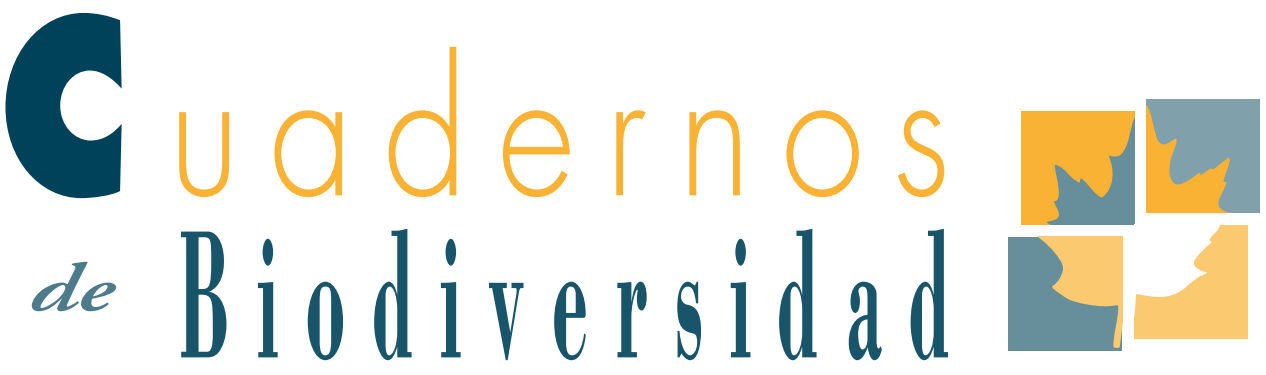

www. cuadernosdebiodiversidad.org

\section{Estado de conservación de Narcissus perezlarae Font Quer (Amaryllidaceae) en la Comunidad Valenciana}

Conservation status of Narcissus perezlarae Font Quer (Amaryllidaceae) in the Vallencian Community

\section{P. Pablo Ferrer-Gallego ${ }^{1,2 *}$, Inmaculada Ferrando ${ }^{1,2}$, Albert} Navarro ${ }^{1,2}$, Josep E. Oltra Benavent ${ }^{1,3}$, Joan Pérez-Botella ${ }^{1,3}$, Roger Carchano ${ }^{1,3}$, Francisco J. Albert ${ }^{1,2}$ \& Emilio Laguna ${ }^{2,3}$

i VAERSA. Avda. Cortes Valencianas 20, 460 i 5 Valencia (España).

2 Generalitat Valenciana, Servicio de Vida Silvestre, Centro para la Investigación y Experimentación Forestal (CIEF). Avda. Comarques del País Valencià i i 4, 46930 Quart de Poblet (Valencia, España).

3 Generalitat Valenciana, Servicio de Vida Silvestre. Complejo Administrativo 9 d’Octubre, Ti, planta 3. Castán Tobeñas, 77. 460 i 8 Valencia.

* FLORA.CIEF@GVA.ES 


\section{RESUMEN}

Narcissus perezlarae está incluido en el Catálogo Valenciano de Especies de Flora Amenazadas de la Comunidad Valenciana (Orden 6/2013) en la categoría "En peligro de extinción". Estudios recientes han demostrado su origen híbrido $(=N$. cavanillesii $\times N$. miniatus $[=N$. serotinus auct. $])$. Por otro lado, el mayor especialista y monografía del género reclamó recientemente su autonomía como especie independiente, como $N$. piifontianus. Esta especie es endémica de la península Ibérica, presente en Alto Alentejo (Portugal), Sevilla y Cádiz (España), y en la parte oriental de la Cordillera Bética, entre Alicante y Valencia. Sin embargo, si se tratara como una especie independiente, sería un endemismo exclusivamente restringido a la Comunidad Valenciana. Siete poblaciones naturales han sido monitoreadas, cuyos datos censales oscilan anualmente. El último censo contiene 1.634 plantas. Desde 2010, se han llevado a cabo cinco experiencias de translocación en la provincia de Alicante. De un total de 8.970 ejemplares introducidos se ha registrado una tasa de supervivencia del $28,4 \%$ (2.545 plantas) en el último censo. Las poblaciones recién creadas aumentan el número de localidades conocidas de la especie en la Comunidad Valenciana y amplían considerablemente su distribución a lo largo del territorio.

Palabras clave: Especies en peligro de extinción, conservación ex situ e in situ, Narcissus, semillas, translocaciones

\section{ABSTRACT}

Narcissus perezlarae is included in the Valencian Catalogue of Threatened Species of the Valencian Community (Order 6/2013) in the category "In danger of extinction". Recent studies have demonstrated its hybridogenic origin $(=N$. cavanillesii $\times$ $N$. miniatus $[=N$. serotinus auct.]). On the other hand, the greatest specialist and monograph of the genus has most recently claimed its autonomy as an independent species, as $N$. piifontianus. This species is endemic to the Iberian Peninsula, present in Alto Alentejo (Portugal), Sevilla and Cadiz
(Spain), and in the eastern part of the Baetic Mountains, between Alicante and Valencia. However, if treated as an independent species, it would be an endemism exclusively restricted to the Valencian Community. Seven natural populations have been monitored, whose census data oscillate annually. The latest census contains 1634 plants. Since 2010, five translocation experiences have been carried out in Alicante province. A total amount of 8,970 plants were translocated and a survival rate of $28,4 \%(2,545$ plants) was observed in the latest census. The newly created populations increase the number of known locations of the species in the Valencian Community and considerably expand their distribution range along the territory.

Key words: Endangered species, ex situ and in situ conservation, Narcissus, seeds, translocations

\section{INTRODUCCIÓN}

La protección y conservación de híbridos es un tema emergente en conservación. Aunque en el pasado se consideró genéricamente que los híbridos eran negativos para la conservación de las especies, y en muchos casos actuales también pueda considerarse así (Rhymer \& Simberloff 1996, Allendorf et al. 2001, Levin 2002), existe un creciente consenso en considerar que la hibridación es uno de los principales motores de la evolución (Arnold 1992, Mallet 2007), y que en algunas ocasiones, los híbridos pueden merecer ser conservados por ser el último reducto del genoma de especies parentales ya extinguidas (Jackiw et al. 2015, Piett et al. 2015). Dentro de la legislación espańola, la de la Comunidad Valenciana es la única que hasta ahora ha integrado conscientemente a los híbridos en el caso de la flora silvestre.

Narcissus perezlarae Font Quer, pro hybr. (narciso de Pérez Lara ) (Amaryllidaceae) (Fig. 1) es una planta incluida en el Catálogo Valenciano de Especies de Flora Amenazadas como "En peligro de extinción" (Anónimo 2009, 2013, Aguilella et al. 2010). Fue descrita como un híbrido entre $N$. cavanillesii Barra \& G. López y $N$. deficiens Herbert, 
y recientes estudios han demostrado su origen hibridógeno producto del cruzamiento entre $N$. cavanillesii y N. obsoletus (Haw.) Steud. [= N. miniatus Donn.-Morg., Koop. \& Zonn.; N. serotinus auct., non L.] (Marques et al., 2010, 2011). No obstante, las poblaciones de la Comunidad Valenciana citadas por Soler (1998) y Fernández Casas (2000) han sido reivindicadas como autónomas y pertenecientes a un taxon independiente con rango de especie, con el nombre de $N$. piifontianus Fern. Casas (Fernández Casas 2009) (Fig. 2), y más concretamente como nomoespecie, es decir, taxon de origen híbrido establecido tras alcanzar la reproducción sexual y su dispersión natural.
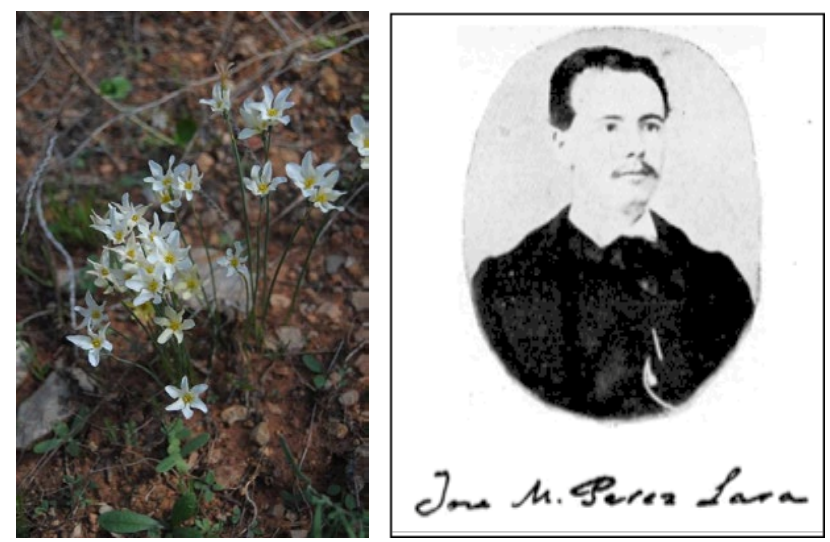

Figura 1. Narcissus perezlarae y retrato y autógrafo José María Pérez Lara publicado por Emilio Fernández Galiano en 1975.
Narcissus perezlarae (incl. N. piifontianus) es un narciso histeranto de floración otoñal, endémico de la península Ibérica, presente al menos en las provincias de Sevilla, Cádiz y en el Alto Alentejo (Portugal), así como en el extremo oriental de las montañas Béticas dentro de la Comunidad Valenciana, en el límite provincial entre Alicante y Valencia (Fernández Casas 2000, Marques et al. 2011) (Fig. 3). En el territorio valenciano se encontró inicialmente por Soler (1998) y con el transcurso del tiempo se han localizado hasta la actualidad seis poblaciones naturales, en los términos de Oliva (Valencia) y Denia, Gata de Gorgos, Ondara y Pego (Alicante). Habita en herbazales y pastizales algo nitrófilos, suelos arcillosos o arenosos más o menos frescos, bordes de caminos y terrenos cultivados (Soler 1998, Aguilella et al. 2010). Varias de sus poblaciones se localizan en terrenos que en la actualidad, o en el pasado reciente, fueron terreno de cultivo, lo que aboga por la posibilidad de que el arado de los campos agrícolas pudiera haber favorecido en algunos casos su expansión por vía vegetativa.
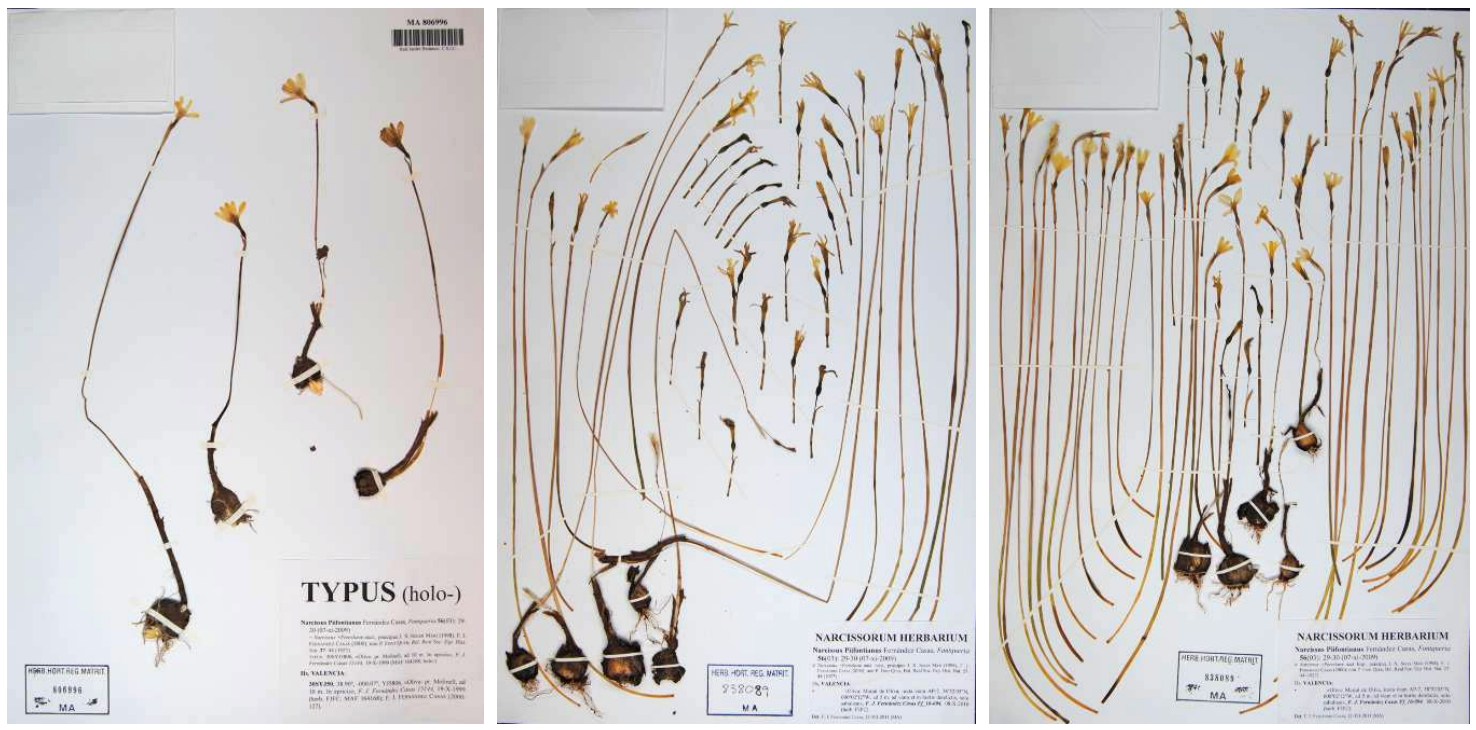

Figura 2. Material utilizado para la descripción de Narcissus piifontianus Fenández Casas procedente de Oliva (pr. Molinell) y recolectado en 1999 y 2010. Foto: Pablo Ferrer. Reproducido con permiso del RJBM. 


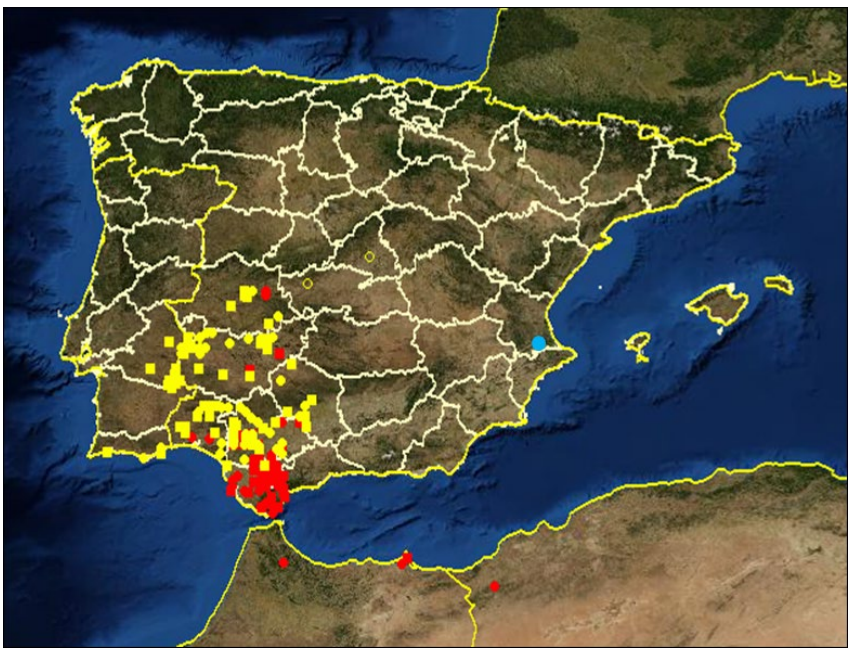

Figura 3. Distribución mundial de los progenitores de Narcissus perezlarae; N. cavanillesii (cuadrado rojo) y $N$. serotinus (cuadrado amarillo) y distribución de $N$. piifontianus en la Comunidad Valenciana.

Un carácter importante, que condiciona el manejo de su germoplasma en los trabajos de conservación ex situ y las posteriores acciones de translocación para la conservación, es que resulta ser una planta prácticamente estéril, o con una producción muy reducida de semillas (Marques et al. 2010), nunca observada en campo hasta el momento en las poblaciones valencianas, ni tampoco en plantas mantenidas en cultivo en condiciones de vivero con material valenciano, salvo por polinización artificial (ver Marques et al. 2010). No obstante, la planta muestra una alta proliferación de bulbos por multiplicación vegetativa, sobre todo cuando es cultivado en condiciones de invernadero, característica que permite una fácil producción de planta en vivero. Esta característica ya fue descrita para híbridos de este grupo por Marqués et al. (2011).

Conforme a Marques et al. (2010) las poblaciones valencianas de esta especie podrían provenir de la generación in situ del híbrido a partir de sus parentales y la posterior extinción de uno de ellos, $N$. cavanillesii. En las áreas donde coincide $N$. obsoletus con los del grupo de $N$. serotinus s.l. ( $N$. serotinus s.str. y $N$. obsoletus) se producen semillas híbridas viables, aunque en proporciones reducidas debido a diversas barreras reproductivas (Marques et al. $2007 \mathrm{a}, \mathrm{b}$ ).
En el presente trabajo se aportan resultados de los censos poblacionales y se detallan las actuaciones de plantación para crear nuevas poblaciones de la especie, siguiendo el concepto de 'neopoblación' descrito por Laguna \& Ferrer-Gallego (2012).

\section{MATERIAL Y MÉTODOS}

\section{Censos}

Los censos poblacionales se han realizado mediante conteo directo de ejemplares adultos. Para las poblaciones naturales se han considerado adultos a las plantas en floración, o con evidencias suficientes de alcanzarla o haberla realizado. Conforme a nuestras observaciones de campo y en vivero, las plantas que alcanzan un mayor diámetro de bulbos -en torno a 1 cm o más- son las que florecen. Los censos de plantas en flor subestiman necesariamente el número real de ejemplares, pero son el único método práctico para obtener datos comparativos, ya que sería imposible conocer el número real en campo por diversas razones, como el carácter críptico de las plantas juveniles, la fácil confusión de los ejemplares sin flor con otras especies de morfología foliar parecida coincidentes en las zonas de muestreo -p. ej., diversas especies de Allium L. o Muscari Mill.-, o la elevada densidad de algunos de los pastizales donde crecen las plantas -los juveniles quedan totalmente ocultos bajo las hojas de plantas de mayor talla y similar coloración foliar.

En el caso de las plantaciones, se han considerado inicialmente como unidades censales los puntos $\mathrm{u}$ hoyos de plantación, ya que por la alta proliferación de bulbos ya indicada, apenas es posible conseguir en cultivo bulbos de diámetros que con seguridad puedan corresponder a plantas adultas. En consecuencia, lo que se planta no son bulbos sueltos sino grupos de ellos obtenidos en una misma maceta $\mathrm{u}$ alveolo de cultivo. Para el seguimiento no se han realizado necesariamente los censos sobre plantas en flor, ya que además de tomarse la situación exacta de cada hoyo mediante GPS convencional en el momento de la plantación, éstos fueron a menudo señalizados; en muchos casos, los autores han observado además que se conserva durante varios años la morfología del hoyo, facilitando la visualización a distancia. 


\section{Producción de planta}

El cultivo de planta se ha realizado en el Centro para la Investigación y Experimentación Forestal de la Generalitat Valenciana (CIEF) siguiendo la metodología expuesta en el trabajo de Ferrer-Gallego et al. (2013) para especies bulbosas. Se han usado dos tipos de contenedores: bandejas tipo QP con alveolos de $200 \mathrm{cc}$. de capacidad de sustrato para el crecimiento inicial, y maceta de $290 \mathrm{cc}$. tras el repicado (Fig. 4). El repicado de los bulbos se ha efectuado a finales de otońo y principios de invierno, ya que conforme a nuestras observaciones, es la mejor época dado el ciclo biológico de este narciso, de floración y crecimiento otoñal. El sustrato estaba compuesto por turba: fibra de coco: perlita, en proporciones 3:1:1. Las plantas alcanzan un estado de desarrollo adecuado para ser plantadas en campo transcurridos 10 meses desde el repicado y separación de los bulbillos de la planta madre; en ese momento el bulbo inicial ya mostraba multiplicación de bulbillos caulinares e incluso la división de éstos como ejemplares independientes. Se ha observado que este método permite una alta cantidad de planta en poco tiempo.

A lo largo de los últimos años se han realizado en el CIEF numerosas experiencias infructuosas de polinización artificial entre $N$. obsoletus y $N$. perezlarae, a fin de obtener semillas y poder conocer el comportamiento de los retrohíbridos.

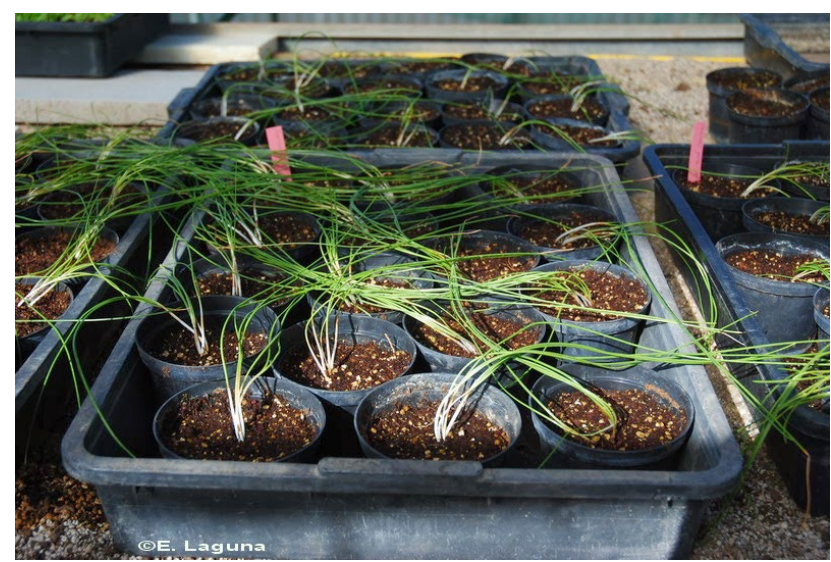

Figura 4. Producción de Narcissus perezlarae en el vivero del CIEF.

\section{Traslocaciones para la conservación}

Se han creado cinco neopoblaciones de $N$. perezlarae en la Comunidad Valencia plantando ejemplares producidos en el vivero del CIEF. Todas las poblaciones se han establecido en el extremo NE de la provincia de Alicante. Dos de ellas se sitúan en un mismo enclave, pero han sido reconocidas con unidades de seguimiento diferentes, ya que las áreas de plantación no se superponen y distan algunos metros entre sí.

\section{RESULTADOS}

\section{Estado de las poblaciones naturales}

Se han localizado 6 poblaciones nativas de la especie, cuyos datos censales se exponen en la tabla 1. Las casillas en blanco indican que ese año no se realizó censo de la población correspondiente, ya que en el programa de monitoreo que desarrolla el CIEF y el servicio de Vida Silvestre de la Generalitat Valenciana, el taxon ha de censarse preferentemente cada 2-3 ańos. De una población -La Marjal, en los municipios de Dénia y El Verger (Alicante)- no se tiene la seguridad de que se conserven efectivos, aunque al descubrirse en 2009 sólo se hallaron 2 ejemplares. Para el resto de localidades, se aprecian importantes fluctuaciones interanuales. El número de adultos en flor censados varía de 0 (Marjal en 2009 y 2016), 2 (id. en 2009) o 5 ejemplares (Peatge AP-7 en 2011) en las poblaciones con menores efectivos, a 1637 (Planisses en 2014) o 2375 (Riu Alberca en 2008) en las que marcan los máximos históricos conocidos. 
Tabla 1. Censo en las poblaciones naturales de Narcissus perezlarae en la Comunidad Valenciana.

\begin{tabular}{l|c|c|c|c|c|c|c|c}
\hline Unidad de seguimiento / años & 2008 & 2009 & 2010 & 2011 & 2012 & 2013 & 2014 & 2016 \\
\hline Partida de Planisses (Gata de Gorgos) & 820 & & 146 & 145 & & & 1637 & 164 \\
\hline Riu Alberca (Dénia) & 2375 & & 96 & 41 & & & 174 & 281 \\
\hline Les Lloberes (Dénia) & & & & & 181 & & 424 & 636 \\
\hline Marjal (El Verger-Dénia) & & 2 & 0 & & & & & 0 \\
\hline El Molinell (Oliva) & 42 & & 439 & 538 & & 79 & 498 & 511 \\
\hline Peatge AP-7 (Ondara) & & & 40 & 5 & 130 & & 22 & 54 \\
\hline Total & 3237 & 2 & 721 & 729 & 311 & 79 & 2755 & 1634
\end{tabular}

Trabajos de traslocación para la conservación

Se indican a continuación datos de las plantaciones realizadas y resultados. Los censos relativos al seguimiento de las poblaciones se aportan en la tabla 2 .

\section{Población Muntanyeta Verda (Parque Natu- ral de la Marjal de Pego-Oliva)}

En 2012 se plantaron 700 ejemplares en 40 puntos u hoyos de plantación. El censo realizado en 2015 mostró 18 puntos con planta (cada uno de ellos con varios individuos). En 2016 no se localizaron ejemplares, aunque por diferentes factores -acumulación de hojarasca de ejemplares próximos de Pinus halepensis Mill. y probable retraso fenológico en ese ańo- conviene esperar a los censos de los siguientes años para comprobar si el intento de establecer una población ha fracasado.

\section{Población El Montgó-Morro Codina (Par- que Natural El Montgó)}

Dentro del Parque Natural de El Montgó -municipios de Dénia y Xàbia, NE de la provincia de Alicante- se han plantado ejemplares en el entorno del área recreativa forestal de Les Planes y cerca del paraje denominado "Morro Codina". Esta última corresponde a calizas y dolomías muy karstificadas y próximas a los grandes acantilados de la zona, que superan los $130 \mathrm{~m}$ de altura, y donde se reciben aparentemente abundante criptoprecipitaciones. La supervivencia en Morro Codina, es del 68,5\% trascurridos cuatro años de la plantación.

\section{Población El Montgó-Les Planes (Parque Natural El Montgó)}

En Les Planes se realizaron dos plantaciones en enclaves cercanos a la zona recreativa del mismo nombre, sobre terrenos horizontales moderadamente karstificados y más alejados del mar, que reciben aparentemente menos criptoprecipitación que la zona citada de Morro Codina; en esta zona la vegetación posee parcialmente una cobertura superior de Pinus halepensis. La primera plantación (Les Planes-1) se hizo en 2010, y el último censo revela una supervivencia del 19,6\% transcurridos 7 ańos, mientras que el núcleo denominado Les Planes-2 tienen una supervivencia del $22,8 \%$ transcurridos 3 años (Tabla 2, Fig. 5).

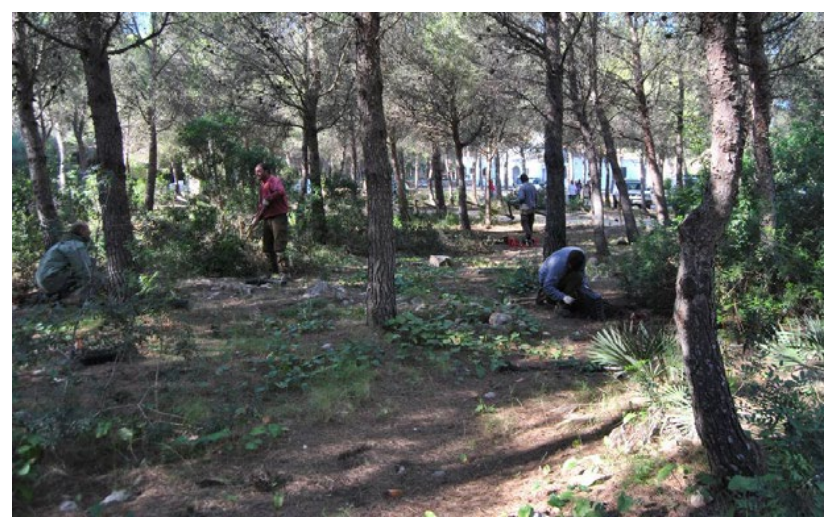

Figura 5. Zona de plantación de Narcissus perezlarae en el LIC y Parque Natural El Montgó, próximo a la zona recreativa de Les Planes. 


\section{Población Vall d'Ebo}

En 2015 se realizó una plantación dentro del Lugar de Interés Comunitario (LIC) Valls de la Marina, en el término municipal de Vall d'Ebo (Alicante). Se introdujeron 1050 plantas en 75 puntos de plantación. Trascurrido un año, el primer censo realizado en octubre de 2016 muestra 770 ejemplares, repartidos en 44 puntos de plantación, de los cuales 25 mostraban floración (Tabla 2, Fig. 6).
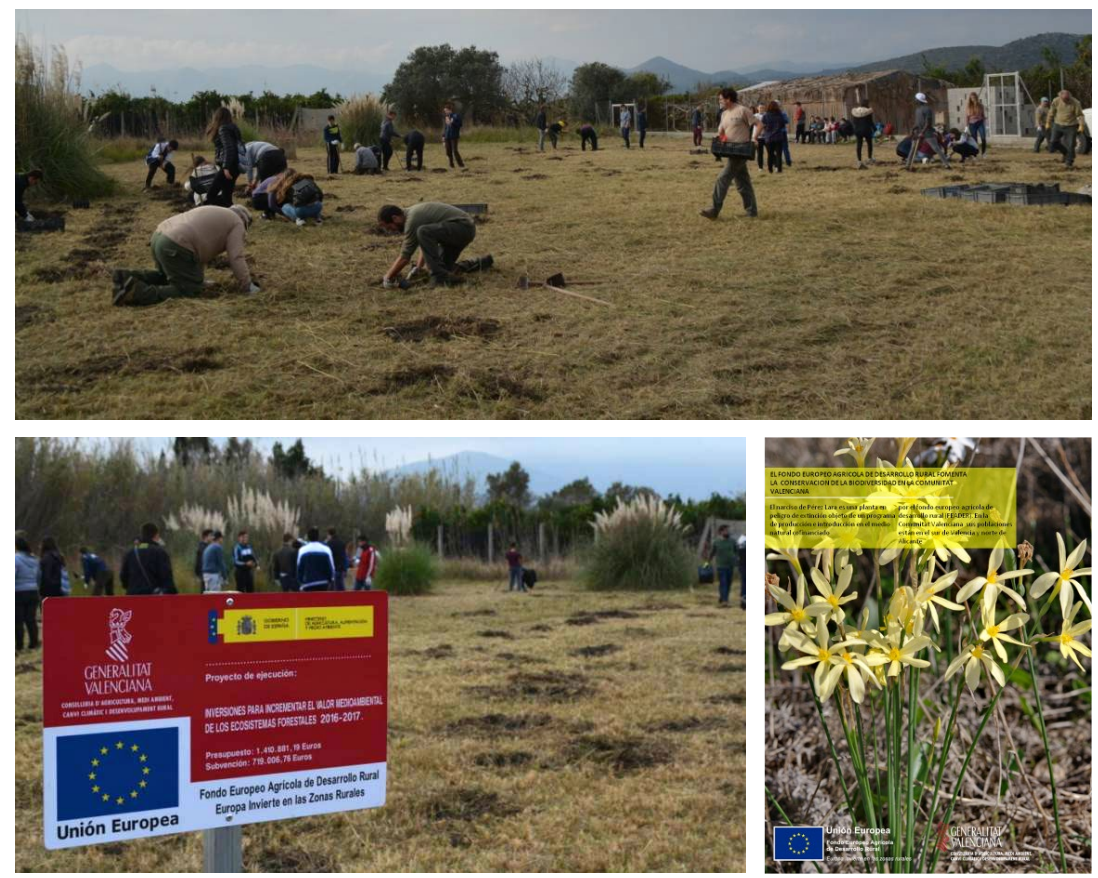

Figura 6. Actividad de participación social; jornada de plantación de Narcissus perezlarae en el Parque Natural PegoOliva, dentro del LIC Marjal de Pego-Oliva con alumnos del IES Gabriel Ciscar.

\section{Población Oliva (Parque Natural de la Marjal de Pego-Oliva)}

A finales de 2016 se ha realizó una plantación en una parcela dentro del municipio de Oliva y del Parque Natural del Marjal de PegoOliva. Se introdujeron 2000 bulbos en 250 hoyos de plantación. Esta actuación se realizó como una actividad de participación social en la que han colaborado el Ayuntamiento de Oliva y el Instituto de Enseñanza Secundaria 'Gabriel Ciscar' (Tabla 2, Fig. 7). El primer censo de este núcleo introducido será realizado en otońo de 2017.

Figura 7. Zona de plantación de Narcissus perezlarae en Vall d'Ebo (LIC Valls de la Marina); ejemplares un año después de su introducción en la zona; primer censo demográfico realizado en 2016 trascurrido un año de la plantación.

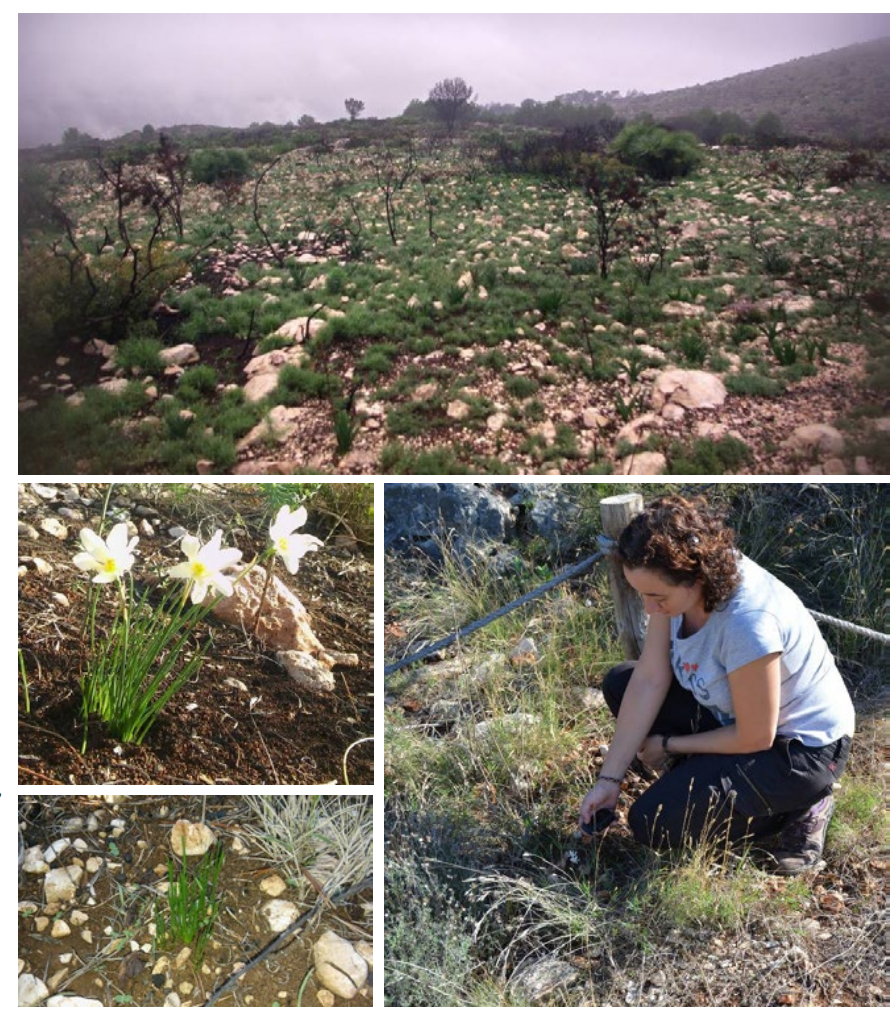


Tabla 2. Plantaciones realizadas con Narcissus perezlarae en la Comunidad Valenciana.

\begin{tabular}{l|c|c|c|c|c}
\multicolumn{1}{c|}{ Población } & $\begin{array}{c}\text { Fecha } \\
\text { plantación }\end{array}$ & $\begin{array}{c}\text { Número } \\
\text { planta } \\
\text { introducida }\end{array}$ & $\begin{array}{c}\text { Año último } \\
\text { censo }\end{array}$ & $\begin{array}{c}\text { Número planta } \\
\text { último censo }\end{array}$ & \% supervivencia \\
\hline Muntanyeta Verda (Pego-Oliva) & $24-1-2012$ & 700 & 2016 & 0 & 0,0 \\
\hline El Montgó-pr. Morro Codina & $18-11-2010$ & 1380 & 2014 & 945 & 68,5 \\
\hline El Montgó-Les Planes 1 & $11-3-2010$ & 1410 & 2017 & 277 & 19,6 \\
\hline El Montgó-Les Planes 2 & $22-11-2013$ & 2430 & 2017 & 553 & 22,8 \\
\hline Vall d'Ebo & $21-10-2015$ & 1050 & 2016 & 770 & 73,3 \\
\hline Oliva (P.N. Pego-Oliva) & $15-12-2016$ & 2000 & - & - & \\
\hline Total (ejemplares plantados) & & 8970 & & & \\
\hline Total & & & & 2545 & 28,4
\end{tabular}

\section{DISCUSIÓN Y CONSIDERACIONES FINALES}

Narcissus perezlarae es un taxon de elevado interés científico debido a la controversia que existe en cuanto a su origen y biología reproductiva. La presencia de las poblaciones valencianas se ha interpretado como resultante de la extinción de uno de los parentales $-N$. cavanillesii- (Marques et al. 2010), lo que justificaría que debe considerarse como una nomoespecie, y que, en consecuencia, la propuesta de abarcarlas bajo un nuevo nombre como el propuesto por Fernández Casas (2009) podría ser consistente. Los intentos fallidos de retrohibridación de las plantas valencianas con $N$. obsoletus en el CIEF, indicadas anteriormente, no apoyan necesariamente ningún aislamiento genético reseñable de la población valenciana, ya que Marques et al. (2010) sí que obtuvieron semillas realizando estos mismos cruzamientos con un elevado número de ejemplares y usando especímenes de este origen geográfico.

La ausencia de fertilidad y producción de semillas, tanto de los ejemplares silvestres como de los cultivados, sólo permite el manejo de germoplasma para la producción de planta en vivero mediante la multiplicación vegetativa de bulbos. Como consecuencia para la conservación, resulta difícil esperar la expansión natural de las poblaciones, ya que la única vía esperable, además de la antropocoria, sería la derivada de intervenciones poco esperables de la fauna silvestre, como el transporte de bulbos. A lo sumo puede preverse que éstos puedan gozar de expansión a muy corta distancia, por ejemplo como consecuencia del hozado del terreno por los jabalíes (Sus scrofa Linnaeus, 1758).

Los resultados de los censos realizados en las diferentes neopoblaciones permiten concluir que se trata de una planta que puede llegar a implantarse bien en el medio natural a partir de ejemplares cultivados, pero la disparidad de los porcentajes de supervivencia encontrados apunta a la necesidad de un conocimiento más detallado de las necesidades ecológicas del taxon; aparentemente las poblaciones con menores tasas de supervivencia podrían haberse implantado en sitios menos aptos, lo que deberá corroborarse en el futuro con un adecuado contraste experimental. Los valores más bajos -Muntanyeta Verda y Les Planes 1 y 2, ver Tabla 2- coinciden con enclaves con una cobertura arbórea laxa o semidensa de Pinus halepensis, ausente en las zonas con porcentajes más elevados, pero igualmente el efecto negativo de esa cobertura, que podría materializarse en una menor resistencia de Narcissus perezlarae a las sustancias alelopáticas producidas por la hojarasca de acículas de pino, no puede aducirse como factor causal en tanto no se hagan contrastes experimentales futuros. 
Las nuevas poblaciones creadas aumentan el número de ejemplares de la especie en la Comunidad Valenciana, y puede hablarse por ahora de un éxito parcial en la estrategia de generar nuevas neopoblaciones, pero de los resultados expuestos se deduce que será necesario abordar en los próximos ańos plantaciones orientadas a contrastar las hipótesis ya apuntadas de la idoneidad de los micrositios de plantación y el efecto de la hojarasca de Pinus halepensis. En cualquier caso, y dada la ausencia casi completa de opciones de dispersión natural, parece evidente que la conservación futura del taxon es altamente dependiente de las acciones de conservación como las aquí indicadas.

\section{AGRADECIMIENTOS}

Gracias a nuestros compañeros del CIEF y del Servicio de Vida Silvestre de la Generalitat Valenciana por su colaboración en los diferentes trabajos que trata este artículo y a los técnicos y personal de los diferentes Parques Naturales en los que se han realizado trabajos de plantación, especialmente a Antoni Pellicer y Manuel Pereira. Las actividades aquí indicadas han sido cofinanciadas por la Comisión Europea a través del programa operativo de los fondos FEOGA-Orientación y FEADER en la Comunidad Valenciana. La producción de Narcissus perezlarae se beneficia del soporte financiero del Fondo Europeo Agrícola de Desarrollo Rural (FEADER). El Fondo Europeo de Desarrollo Rural fomenta la biodiversidad en la Comunidad Valenciana.

\section{REFERENCIAS}

Aguilella, A., Fos, S. \& Laguna, E. (eds.) (2010). Catálogo Valenciano de Especies de Flora Amenazadas. Colección Biodiversidad, 18. Conselleria de Medi Ambient, Aigua, Urbanisme i Habitatge, Generalitat Valenciana, Valencia, 358 pp.

Allendorf, F.W., Leary, R.F., Spruell, P. \& Wenburg, J.K. (2001). The problems with hybrids: setting conservation guidelines. Trends in Ecology and Evolution, 16:613-622.
Anónimo (2009). Decreto 70/2009, de 22 de mayo, del Consell, por el que se crea y regula el Catálogo Valenciano de Especies de Flora Amenazadas y se regulan medidas adicionales de conservación. Diari Oficial de la Comunitat Valenciana, 6021:20143-20162.

Anónimo (2013). Orden 6/2013, de 25 de marzo, de la Conselleria de Infraestructuras, Territorio y Medio Ambiente, por la que se modifican los listados valencianos de especies protegidas de flora y fauna. Diari Oficial de la Comunitat Valenciana, 6996:8682-8690.

Arnold, M.L. (1992). Natural hybridization as an evolutionary process. Annual Review of Ecology, Evolution and Systematics, 23:237-261.

Fernández Casas, F.J. (2000). Narcissorum notulae, XIII. Fontqueria, 56(3):29-30.

Fernández Casas, F.J. (2009). Narcissorum notulae, V. Fontqueria, 54(6):123-128.

Ferrer-Gallego, P.P., Ferrando, I., Gago, C. \& Laguna, E. (eds.), (2013). Manual para la conservación de germoplasma y el cultivo de la flora valenciana amenazada. Colección Manuales Técnicos Biodiversidad, 3. Conselleria d'Infraestructures, Territori i Medi Ambient, Generalitat Valenciana, Valencia, 246 pp.

Jackiw, R.A., Mandil, G. \& Hager, H. (2015). A framework to guide the conservation of species hybrids based on ethical and ecological considerations. Conservation Biology, 29:1040-1051.

Laguna, E. \& Ferrer-Gallego, P.P. (2012). Reforzamientos de proximidad y Neopoblaciones de seguridad, nuevos conceptos complementarios para determinados tipos $\mathrm{d} e$ implantaciones vegetales in situ. Conservación Vegetal, 16:14.

Levin, D.A. (2002). Hybridization and extinction: in protecting rare species, conservationists should considerer the dangers of inbreeding, which compound the more wellknown threats to wildlife. American Scientist, 90:254-259.

Mallet, J. (2007). Hybrid speciation. Nature 446: 279-283.

Marques, I., Rosselló-Graell, A., Draper, D. \& Iriondo, J.M. (2007a). Pollination patterns limit hybridization between two sympatric species in Narcissus (Amaryllidaceae). American Journal of Botany, 94(8):1352-1359. 
Marques, I., Rosselló-Graell, A., Draper, D. \& Iriondo, J.M. (2007b). Pollination ecology and hybridization between Narcissus cavanillesii A. Barra \& G. López and N. serotinus L. in Portugal. Bocconea, 21:65-75

Marques, I., Nieto, G., Draper, D., Martins-Loução, M.A. \& Fuertes, J. (2010). Unraveling cryptic reticulate relationships and the origin of orphan hybrid disjunct populations in Narcissus. Evolution, 64(8):2353-2368.

Marques I, Nieto G, Martins-Loução MA \& Fuertes J. (2011). Fitness in Narcissus hybrids: low fertility is overcome by early hybrid vigour, absence of exogenous selection and high bulb propagation. Journal of Ecology, 99:1508-1519.
Piett, S., Hager, H.A. \& Gerrard, C. (2015). Characteristics for evaluating the conservation value of species hybrids. Biodiversity and Conservation, 24:1931-1955.

Rhymer, J.M. \& Simberloff, D. (1996). Extinction by hybridization and introgression. Annual Review of Ecology, Evolution and Systematics, 27:83-109.

Soler, J.X. (1998). Descubrimiento de Narcissus perezlarae Font Quer (Amaryllidaceae) en el levante español. Anales del Jardín Botánico de Madrid, 56(1):165-166. 
Este número ha recibido una ayuda del Vicerrectorado de Investigación y Transferencia de Conocimiento de la Universidad de Alicante
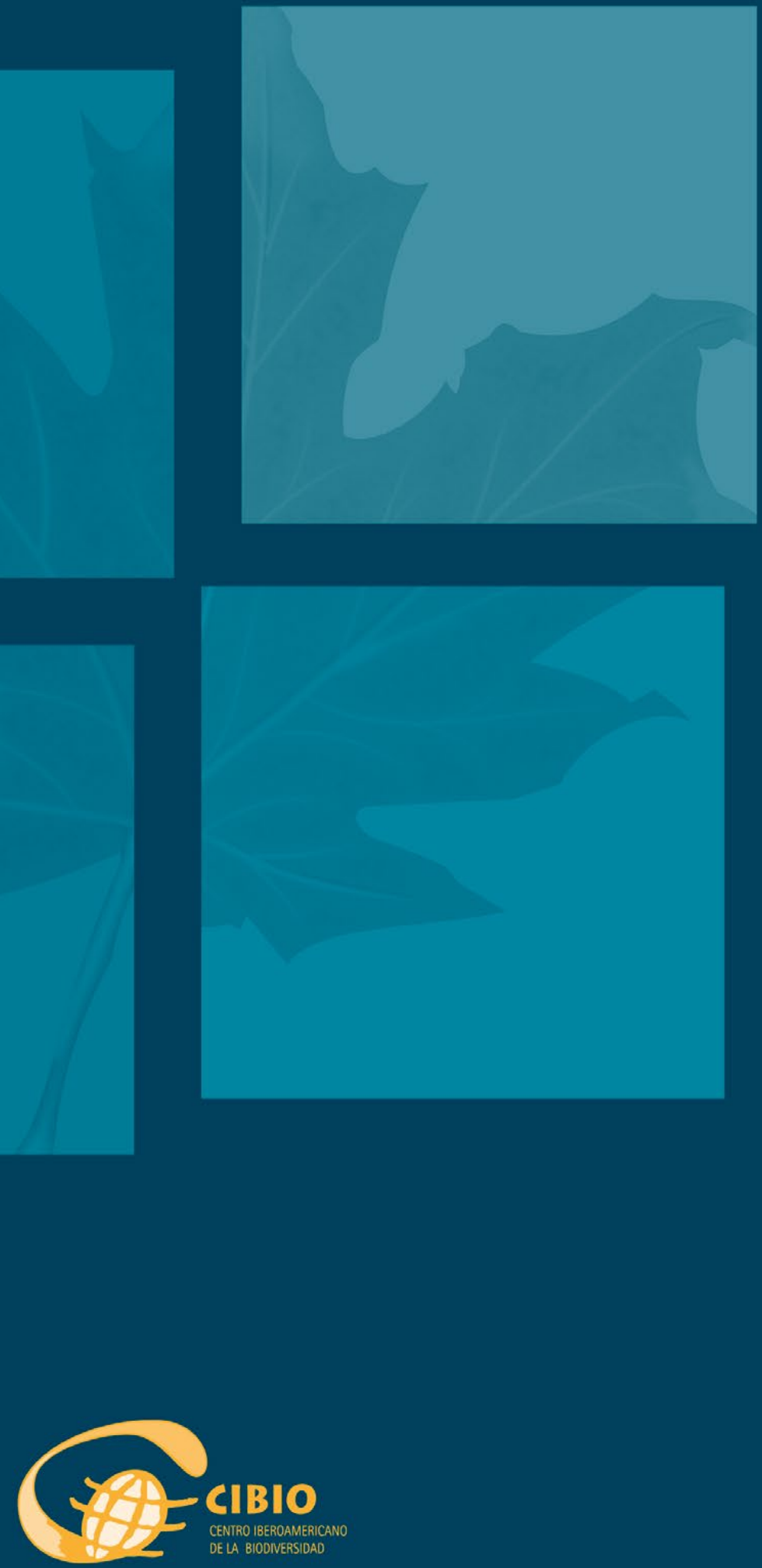

\section{Universitat d'Alacant
Universidad de Alicante}

